\title{
Comportamento de genótipos de soja submetidos a déficit hídrico intenso em casa de vegetação
}

\author{
Samuel Luiz Fioreze ${ }^{1}$, Laerte Gustavo Pivettal, Ademir Fano², Fábio Ribeiro Machado², \\ Vandeir Francisco Guimarães ${ }^{3}$
}

\begin{abstract}
RESUMO
O presente estudo teve por objetivo avaliar o comportamento e as características envolvidas nas respostas de três genótipos de soja, submetidos à indução de déficit hídrico de alta intensidade em casa de vegetação. A pesquisa foi conduzida na Estação de Horticultura e Controle Biológico Professor Mario César Lopes, NEE/UNIOESTE, Marechal Cândido Rondon, Paraná, no ano de 2007. Utilizou-se um esquema fatorial 3x2, sendo três genótipos de soja (CD 201, CD 202 e CD 217) e dois regimes hídricos (irrigação satisfatória e déficit hídrico), em delineamento inteiramente casualizado, com cinco repetições. O déficit hídrico foi imposto por meio de suspensão da rega, no início da fase reprodutiva da cultura. Foram avaliados a umidade gravimétrica do solo (Ug) e o conteúdo relativo de água nas folhas (CRA), durante o período de déficit hídrico; a liberação de eletrólitos de discos foliares, no momento da reidratação; as variáveis biométricas, no final do período de recuperação e os componentes da produção e produção por planta, no final do ciclo da cultura. O déficit hídrico aplicado afetou de maneira distinta os genótipos testados, quanto à manutenção do status hídrico, da área foliar e, por consequência, do potencial produtivo. A diferenciação no comportamento dos genótipos foi possível, mesmo em condições de déficit hídrico intenso, sendo o CD 202 o que apresentou melhor comportamento. A manutenção do CRA e da área foliar devem ser consideradas como características de interesse no desenvolvimento de materiais adaptados a condições de restrição hídrica.
\end{abstract}

Palavras-chave: Glycine max (L.) Merr., disponibilidade hídrica, conteúdo relativo de água, área foliar, tolerância protoplasmática.

\section{ABSTRACT}

\section{Performance of soybean genotypes under high intensity drought stress in greenhouse conditions}

The objective of this study was to evaluate the performance and the traits involved in the responses of three soybean genotypes to high intensity drought stress under greenhouse conditions. The experiment was carried out at the Greenhouse and Horticulture Station Professor Mario César Lopes, NEE/UNIOESTE - Marechal Cândido Rondon, PR, Brazil, in 2007. Were evaluated three soybean genotypes (CD 201, CD 202 and CD 217) subjected to a high intensity and short drought stress period. The experiment was arranged in a (3x2) factorial completely randomized design with five replications. Drought stress was induced at the beginning of the reproductive stage by watering suspension. Soil moisture content $(\mathrm{Ug})$ and relative leaf water content (RWC) were measured during drought stress and rehydration period. Protoplasmic tolerance was evaluated before rehydration and the biometric parameters were determined after

\footnotetext{
Recebido para publicação em março de 2010 e aprovado em março de 2011

${ }^{1}$ Engenheiros-Agrônomos, Mestres. Departamento de Produção Vegetal, Universidade Estadual Paulista, UNESP, Faculdade de Ciências Agronômicas de Botucatu, Fazenda Experimental Lageado, s/n, 18603-970, Botucatu, São Paulo, Brasil. slfioreze@ @fca.unesp.br (autor para correspondência); lgpivetta@fca.unesp.br

${ }^{2}$ Engenheiros-Agrônomos. Departamento de Agricultura, Universidade Estadual do Oeste do Paraná, Rua Pernambuco, n ${ }^{\circ}$ 1777, Caixa Postal 1008, Centro, 85960-000, Marechal Cândido Rondon, Paraná, Brasil. ademirfano@yahoo.com.br; fabio_rmachado@yahoo.com.br

${ }^{3}$ Engenheiro-Agrônomo, Doutor. Departamento de Agricultura, Universidade Estadual do Oeste do Paraná, Rua Pernambuco, n ${ }^{\circ} 1777$, Caixa Postal 1008, Centro, 85960-000, Marechal Cândido Rondon, Paraná, Brasil. vandeirfg@yahoo.com.br
}

Rev. Ceres, Viçosa, v. 58, n.3, p. 342-349, mai/jun, 2011 
the recovery period. Yield and yield components were determined at the end of crop cycle. Soybean genotypes were differently affected by drought stress regarding water status and leaf area maintenance, and consequently the yield potential. The differences among the soybean genotypes could be verified even at high drought stress intensity, with CD 202 showing the best performance. Water status and leaf area maintenance must be considered as characteristics of interest for drought tolerance improvement in soybean breeding programs.

Key words: Glycine max L. (Merrill), leaf area, leaf protoplasmic tolerance, relative water content, soil water.

\section{INTRODUÇÃO}

A disponibilidade hídrica é considerada o fator climático de maior efeito sobre a produtividade agrícola, sendo o fator que rege a distribuição das espécies nas diferentes zonas climáticas do globo (Lawlor \& Uprety, 1993). A produtividade de plantas cultivadas em ambientes com restrição hídrica é, frequentemente, afetada por características da planta que permitem a manutenção do status hídrico durante o dessecamento do solo (Blum, 2005).

Existem duas estratégias básicas pelas quais as plantas resistem à seca: o "evitamento" e a tolerância à desidratação (Levit, 1972). Características de "evitamento" à desidratação atuam na manutenção do conteúdo relativo de água (CRA) em tecidos, durante o período de déficit hídrico. Plantas que apresentam a estratégia de tolerância apresentam tecidos que podem tolerar a desidratação, até certo ponto (baixo conteúdo relativo de água crítico) e, frequentemente, apresentam ajuste osmótico. A estratégia de tolerância permite às plantas manter a atividade metabólica com a evolução do déficit hídrico e declínio do CRA (Lawn \& Likoswe, 2008). A cultura da soja apresenta tanto características de "evitamento" como de "tolerância” à desidratação (Sinclair \& Ludlow, 1986).

A dificuldade na identificação de um parâmetro fisiológico confiável como indicador de produtividade em condição de seca tem sugerido que a performance produtiva, em uma série de ambientes, deveria ser utilizada como o principal indicador para tolerância à seca (Voltas et al., 2005). Pode-se esperar que não exista um padrão similar de respostas que seja altamente correlacionado com a produtividade, em todos os ambientes com déficit hídrico (Cattivelli et al., 2008). Um correto entendimento de como o déficit hídrico afeta o desenvolvimento das culturas seria de grande ajuda para a criação de mais cultivares tolerantes à seca (Turner, 1997), por meio da identificação de características especificas, que determinam a performance de culturas em condições de déficit hídrico e que são passíveis de alterações, seja por transformação genética, seja por melhoramento genético convencional.

Várias características têm sido relacionadas com a tolerância à seca em plantas cultivadas. A utilização de métodos simples, como a determinação da umidade gravimétrica do solo (Ug) e o conteúdo relativo de água
(CRA) são apontados como eficientes no estudo da adaptação de plantas à seca, bem como na comparação de materiais em programas de melhoramento (screening) (Jones, 2007). A manutenção do CRA tem sido eficiente na diferenciação de genótipos de soja, em grande parte dos trabalhos de pesquisa relacionados com déficit hídrico, apontando elevada correlação com a manutenção da área foliar (James et al., 2008b) e com o ajuste osmótico (James et al., 2008a). O CRA tem apresentado também, relação linear com os valores de potencial hídrico foliar, em condições de déficit hídrico suave, moderado e severo (Ennahli \& Earl, 2005).

Neste contexto, o presente estudo teve por objetivo avaliar o comportamento e as características envolvidas nas respostas de três genótipos de soja, submetidos à indução de déficit hídrico de alta intensidade, em casa de vegetação.

\section{MATERIAL E MÉTODOS}

O experimento foi conduzido no período de janeiro a abril do ano de 2007, em cultivo protegido, na Estação de Horticultura e Controle Biológico Professor Mário César Lopes, pertencente ao Núcleo de Estações Experimentais da Universidade Estadual do Oeste do Paraná UNIOESTE, Campus de Marechal Cândido Rondon, Paraná. A estação está localizada nas coordenadas $54^{\circ} 22^{\prime}$ W longitude, latitude $24^{\circ} 46^{\prime} \mathrm{S}$ e altitude de $420 \mathrm{~m}$. O solo utilizado no experimento foi coletado no horizonte A de um Latossolo Vermelho eutroférrico (EMBRAPA, 2006) com textura argilosa $\left(629,0 \mathrm{~g} \mathrm{~kg}^{-1}\right.$ de argila), que apresentava: 17,7 $\mathrm{g} \mathrm{dm}^{-3}$ de matéria orgânica; $\mathrm{pH}(\mathrm{CaCl})$ 5,6; 2,37 $\mathrm{cmol}_{\mathrm{c}} \mathrm{dm}^{-3} \mathrm{de} \mathrm{Ca} ; 1,5 \mathrm{cmol} \mathrm{dm}^{-3} \mathrm{de} \mathrm{Mg} ; 0,0 \mathrm{cmol} \mathrm{dm}_{\mathrm{c}} \mathrm{de}^{-3} \mathrm{de}$ $\mathrm{Al} ; 0,70 \mathrm{cmol}_{\mathrm{c}} \mathrm{dm}^{-3} \mathrm{de} \mathrm{K} ; 33,89 \mathrm{mg} \mathrm{dm}^{-3}$ de P (Mehlich-1); e V $(\%)$ de 49,65.

O experimento foi conduzido em esquema fatorial $3 \mathrm{X} 2$, em delineamento experimental inteiramente casualizado, com cinco repetições. Os tratamentos foram compostos pela combinação de três genótipos de soja (CD 201, CD 202 e CD 217) e dois regimes hídricos (irrigado, e com imposição de déficit hídrico no início da fase reprodutiva). Os genótipos foram escolhidos dentro de um mesmo programa de melhoramento, de acordo com a sua adaptabilidade à região de cultivo. 
A parcela experimental foi composta por um vaso de polietileno $(8,0 \mathrm{~L})$ com duas plantas de soja. O experimento teve um total de 90 vasos, sendo $1 / 3$ para as coletas de material, durante a imposição do déficit hídrico, 1/3 para a análise biométrica, no final do período de déficit e, o restante, para a avaliação dos componentes da produção e produção por vaso, ao final do ciclo da cultura.

A semeadura dos genótipos de soja foi realizada de maneira escalonada, a fim de sincronizar o período de florescimento, no início das avaliações. Os vasos foram preenchidos com $8 \mathrm{dm}^{3}$ de solo seco e adubado com 687 $\mathrm{mg} \mathrm{dm}^{-3} \mathrm{de}_{2} \mathrm{O}_{5}$ (super fosfato simples) e $78 \mathrm{mg} \mathrm{dm}^{-3} \mathrm{de}$ $\mathrm{K}_{2} \mathrm{O}$ (cloreto de potássio). Antes da semeadura, as sementes foram tratadas com fungicida (metalaxyl-m) e inseticida (imidacloprid). No momento da semeadura, as sementes receberam tratamento com cobalto e molibdênio e, em seguida, foram inoculadas com bactérias do gênero Rhizobium, a fim de estimular a formação de nódulos e a fixação biológica de nitrogênio.

O suprimento de água às plantas foi realizado de modo a manter a umidade do solo próxima à capacidade de campo, até o início do período de florescimento (estádio R1), quando a imposição do déficit hídrico foi realizada. O déficit hídrico foi aplicado por meio de suspensão da rega,

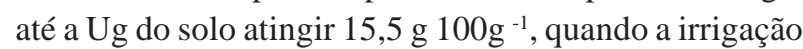
foi retomada. $\mathrm{O}$ método de suspensão da rega, juntamente com a elevada demanda evapotranspiratória do ambiente de cultivo, permitiu o desenvolvimento de um déficit hídrico de alta intensidade e curta duração. Após a reidratação, todos os tratamentos foram conduzidos com suprimento adequado de água, até o final do ciclo da cultura.

Durante o período de imposição do déficit hídrico e recuperação das plantas, foi determinado o CRA diariamente, antes do nascer do sol, segundo metodologia proposta por Barrs \& Weatherley (1962), por meio da pesagem das massas de matéria fresca (MF), túrgida (MT) e seca (MS), de discos foliares, utilizando-se a fórmula: $\mathrm{CRA}=$ (MF-MS)/ (MT-MS) x $100 \%$. A tolerância protoplasmática à dessecação foliar foi avaliada no último dia de déficit hídrico, mediante a determinação da condutividade elétrica resultante da liberação de eletrólitos de discos de foliares imersos em $30 \mathrm{~mL}$ de água deionizada (Leopold et al., 1981).

Após o período de recuperação das plantas submetidas ao déficit hídrico, foram determinadas as variáveis biométricas: área foliar, massa da matéria seca de folhas, caules + pecíolos e estruturas reprodutivas. No final do ciclo da cultura (estádio R8), foram avaliados os componentes da produção: número total de vagens por planta, número de vagens viáveis por planta, número de grãos por vagem, número de grãos por planta, massa de grãos por planta e massa de mil grãos.
Os dados foram submetidos à análise de variância pelo teste $\mathrm{F}$ e, quando detectadas variações significativas, as médias foram contrastadas pelo teste de Tukey a 5\% de significância. Os valores de CRA e Ug foram submetidos ao teste de correlação de Pearson.

\section{RESULTADOS E DISCUSSÃO}

O resultado da análise de variância demonstrou a existência de interações significativas, entre genótipos e regimes hídricos, para todas as variáveis determinadas, com exceção da matéria seca de estruturas reprodutivas, número de grãos por vagem e massa de mil grãos (Tabelas 1 e 2).

A Ug do solo permaneceu constante, durante o período de déficit hídrico e recuperação para os tratamentos irrigados (Figura 1), enquanto, para os tratamentos submetidos ao déficit hídrico, observou-se queda acentuada nos três primeiros dias, com pouca variação entre o terceiro e o quarto dia, no momento da reidratação. O padrão de dessecamento do solo permitiu a imposição de um déficit hídrico de alta intensidade e curta duração, em função das condições do ambiente de cultivo, com temperaturas máximas de $40,5^{\circ} \mathrm{C}$, favorecendo um déficit de pressão de vapor atmosférico.

Todos os genótipos submetidos a déficit hídrico apresentaram redução do CRA, em comparação com os tratamentos mantidos sob irrigação (Figura 2). Entretanto, o genótipo CD 217 mostrou-se mais sensível ao dessecamento do solo, apresentando os menores valores de CRA durante o período de déficit hídrico e reidratação. Este comportamento parece estar associado ao menor controle estomático das perdas de água, em resposta à diminuição da disponibilidade de água no solo. Em decorrência da sensibilidade observada no genótipo CD 217, observou-se um maior tempo de recuperação do CRA após a reidratação. Os genótipos CD 201 e CD 202 apresentaram comportamento semelhante entre si, tanto para o período de déficit hídrico, quanto após a reidratação, apresentando rápida recuperação.

Em condições de déficit hídrico suave, moderado e severo, Ennahli \& Earl (2005) observaram uma relação linear entre os valores de conteúdo relativo de água (CRA) e potencial hídrico foliar, em plantas de algodão. James et al. (2008a) observaram variações genéticas, dentro de grupos de cultivares e espécies selvagens de soja, para características de interesse no estudo da tolerância à seca em plantas, como CRA e potencial hídrico, tendo encontrado o CRA como um bom covariante para determinação de variações genéticas, nos valores de ajuste osmótico em folhas de soja. Pannu et al. (1993) observaram que a manutenção da turgescência foliar foi de grande utilidade no screening para tolerância à seca em grão de bico. 
Na Figura 3, é apresentada a correlação existente entre os valores de Ug do solo e CRA, para o período de déficit hídrico, onde é possível observar o padrão de desidratação foliar, em função do dessecamento do solo. $\mathrm{O}$ maior coeficiente de correlação, observado para o genótipo CD 217, indica sua maior sensibilidade à diminuição da água disponível no solo, quando comparado com os demais genótipos. Sinclair \& Ludlow (1986) sugeriram que, em condições de déficit hídrico severo, em que os estômatos permanecem fechados na maior parte do dia, os baixos valores de condutância estomática são de grande importância para a manutenção do CRA; portanto, a sensibilidade dos estômatos ao abaixamento da umidade do solo torna-se um comportamento desejável no desenvolvimento de novos genótipos mais tolerantes a cultivos sob déficit hídrico, que apresentem uma rápida retomada da atividade fotossintética após a reidratação.

A comparação dos genótipos quanto ao extravasamento de eletrólitos celulares (Figura 4) mostra, claramente, os efeitos da redução do conteúdo de água nas folhas de soja, em condição de déficit hídrico intenso. O maior grau de desidratação foliar apresentado pelo genótipo CD 217, no momento da reidratação, refletiu-se em maiores valores de condutividade elétrica da solução. Os genótipos CD 201 e CD 202 apresentaram um padrão de comportamento semelhante ao demonstrado para o CRA, com valores de condutividade próximos aos tratamentos irrigados.

A liberação de eletrólitos ocorre em níveis severos de déficit hídrico, por causa do aumento da quantidade de espécies ativas de oxigênio (superóxidos), radicais livres e de enzimas de "lise" (Roy-Macauley et al., 1992), que resultam na ruptura e aumento da permeabilidade das membranas e, muitas vezes, em danos irreversíveis nas organelas e moléculas presentes no interior das células (Alonso et al., 1997). Plantas de feijão, submetidas a déficit hídrico em casa de vegetação, também apresentaram aumento da liberação de eletrólitos em água deionizada (Pimentel et al., 2000). A tolerância protoplasmática à desidratação foliar foi observada, também, em genótipos de feijão caupi (Pimentel et al., 2002) e grão de bico (Gupta et

Tabela 1. Resumo da análise de variância para relações hídricas de três genótipos de soja sob irrigação e com indução de déficit hídrico no início do período reprodutivo da cultura

\begin{tabular}{lcccccc}
\hline \multirow{2}{*}{ FV $^{1}$} & \multicolumn{7}{c}{ Conteúdo relativo de água nas folhas } \\
\cline { 2 - 7 } & 1 DAI $^{2}$ & 2 DAI & 3 DAI & 4 DAI & 5 DAI & 6 DAI \\
\hline F Genótipo & 5,65 & 5,65 & 5,65 & 5,65 & 5,65 & 5,65 \\
F Regime & 9,89 & 9,89 & 9,89 & 9,89 & 9,89 & 9,89 \\
F G x R & 3,28 & 3,28 & 3,28 & 3,28 & 3,28 & 3,28 \\
CV $(\%)$ & 3,35 & 3,81 & 7,62 & 11,89 & 19,65 & 4,13 \\
\hline
\end{tabular}

\begin{tabular}{lccrr}
\hline \multirow{2}{*}{ FV } & \multicolumn{3}{c}{ Tolerância protoplasmática } \\
\cline { 2 - 5 } & 1 HAC $^{3}$ & 2 HAC & 3 HAC & 4 HAC \\
\hline F Genótipo & 2,97 & 2,82 & 3,19 & 3,83 \\
F Regime & $18,83^{* *}$ & $18,73^{* *}$ & 18,86 & $18,48^{* *}$ \\
F G x R & 3,01 & 2,63 & 3,15 & 3,96 \\
CV $(\%)$ & 71,04 & 72,38 & 74,39 & 74,59 \\
\hline
\end{tabular}

${ }^{1} \mathrm{FV}$ : fonte de variação; ${ }^{2} \mathrm{DAI}$ : dias após o início do déficit hídrico; ${ }^{3}$ HAC: horas após a coleta; ${ }^{\text {ns }}$ não significativo; $*$ e $* *$ significativo a 5 e $1 \%$ de probabilidade, respectivamente, pelo teste $\mathrm{F}$.

Tabela 2. Resumo da análise de variância para variáveis biométricas e componentes da produção de três genótipos de soja sob irrigação e com indução de déficit hídrico no início do período reprodutivo da cultura

\begin{tabular}{lcccccc}
\hline FV $^{1}$ & AF & MSF & MSCP & MSER & MSPA & MST \\
\hline F Genótipo & $22,28^{* *}$ & $10,80^{* *}$ & $63,92^{* *}$ & $25,94^{* *}$ & $46,98^{* *}$ & $28,10^{* *}$ \\
F Regime & $20,43^{* *}$ & $62,28^{* *}$ & $33,53^{* *}$ & $1,81^{\mathrm{ns}}$ & $50,70^{* *}$ & $36,78^{* *}$ \\
F (G x R) & $11,49^{* *}$ & $14,20^{* *}$ & $6,75^{* *}$ & $1,03^{\mathrm{ns}}$ & $11,07^{* *}$ & $7,51^{* *}$ \\
CV $(\%)$ & 12,57 & 10,19 & 8,06 & 26,46 & 8,10 & 8,90 \\
\hline FV & NVTP & NVVP & NGPV & NGPP & MGP & MMG \\
\hline F Genótipo & $10,12^{* *}$ & $5,02^{*}$ & $24,37^{* *}$ & $7,06^{* *}$ & $7,28^{* *}$ & $8,45^{* *}$ \\
F Regime & $27,75^{* *}$ & $10,11^{* *}$ & $4,66^{*}$ & $3,99 \mathrm{~ns}$ & $39,32^{* *}$ & $31,57^{* *}$ \\
F (G x R) & $4,74^{*}$ & $7,17^{* *}$ & $2,03^{\mathrm{ns}}$ & $5,91^{* *}$ & $10,82^{* *}$ & $0,50^{\text {ns }}$ \\
CV $(\%)$ & 15,37 & 19,21 & 18,88 & 19,63 & 15,32 & 10,66 \\
\hline
\end{tabular}

${ }^{1} \mathrm{FV}$ : fonte de variação; AF: área foliar; MSF: massa de matéria seca das folhas; MSCP: massa de matéria seca do caule e pecíolo; MSER: massa de matéria seca de estruturas reprodutivas; MSPA: massa de matéria seca da parte aérea; MST: massa de matéria seca total; NVTP: número total de vagens por planta; NVVP: número de vagens viáveis por planta; NGPV: número de grãos por vagem; NGPP: número de grãos por planta; MGP: massa de grãos por planta; MMG: massa de mil grãos; ns não significativo; * e ** significativo a 5 e $1 \%$ de probabilidade, respectivamente, pelo teste $\mathrm{F}$. 
al., 2000), quando genótipos tolerantes apresentaram baixo índice de injúrias nas membranas.

Para as variáveis biométricas (Tabela 3), determinadas após o período de recuperação das plantas, o genótipo CD 217 apresentou os maiores valores de área foliar e acúmulo de matéria seca, quando comparado com os demais genótipos em cultivo irrigado. Quando submetidos a déficit hídrico, os genótipos CD 201 e CD 217 apresentaram significativa redução nos valores de matéria seca. O genótipo CD 217, que apresentou os maiores valores de área foliar em condição irrigada, apresentou drástica redução quando submetido a déficit hídrico, em função da elevada superfície de perda de água por transpiração. O genótipo CD 202 manteve valores constantes de área foliar

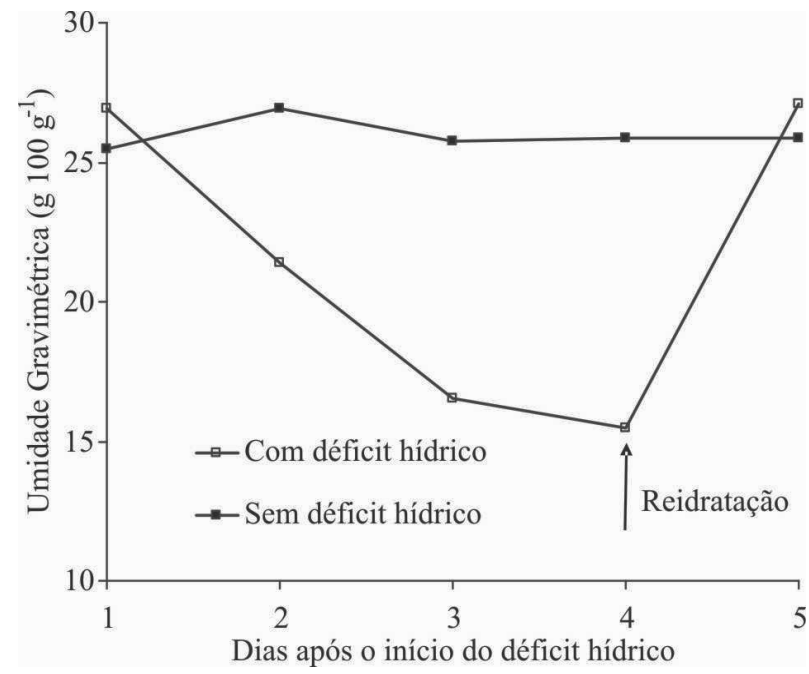

Figura 1. Umidade gravimétrica do solo no período de déficit hídrico e recuperação de plantas de soja.

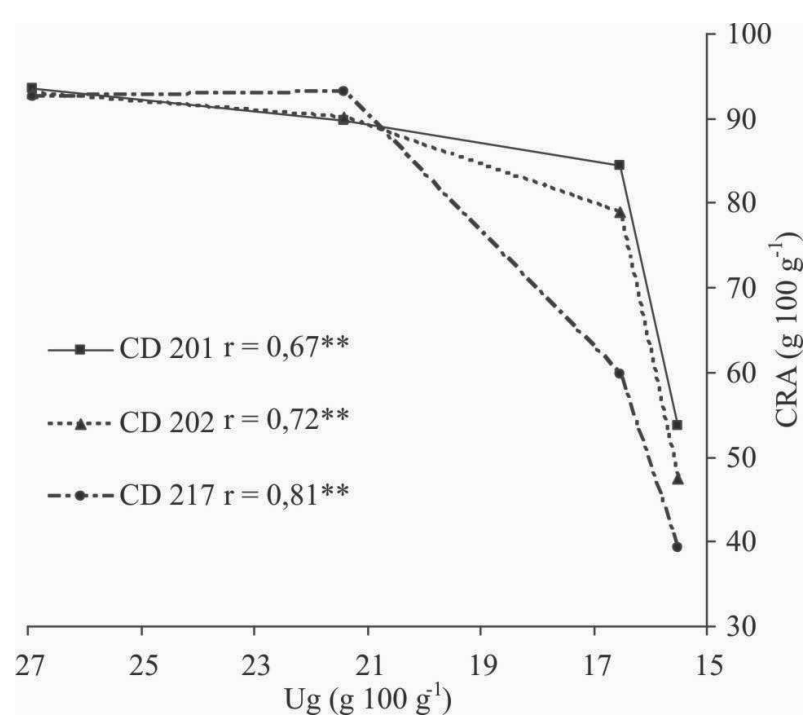

Figura 3. Correlação de Pearson para Ug solo e o CRA no "pre dawn" de genótipos de soja, submetidos a déficit hídrico intenso no início do período reprodutivo. "** significativo a $1 \%$ de probabilidade. e acúmulo de massa de matéria seca, independentemente do sistema de cultivo.

Um dos primeiros processos afetados, em resposta à diminuição da água disponível no solo, é a expansão celular, processo altamente dependente da turgescência da planta. Em estágios mais avançados de déficit hídrico, outros processos fisiológicos são afetados, com efeitos diretos no acúmulo de assimilados pela planta, como a redução na taxa de assimilação de carbono e aumento da taxa respiratória (Turner, 1997). A manutenção de área foliar, para os genótipos CD 201 e CD 202, em condição de défi-

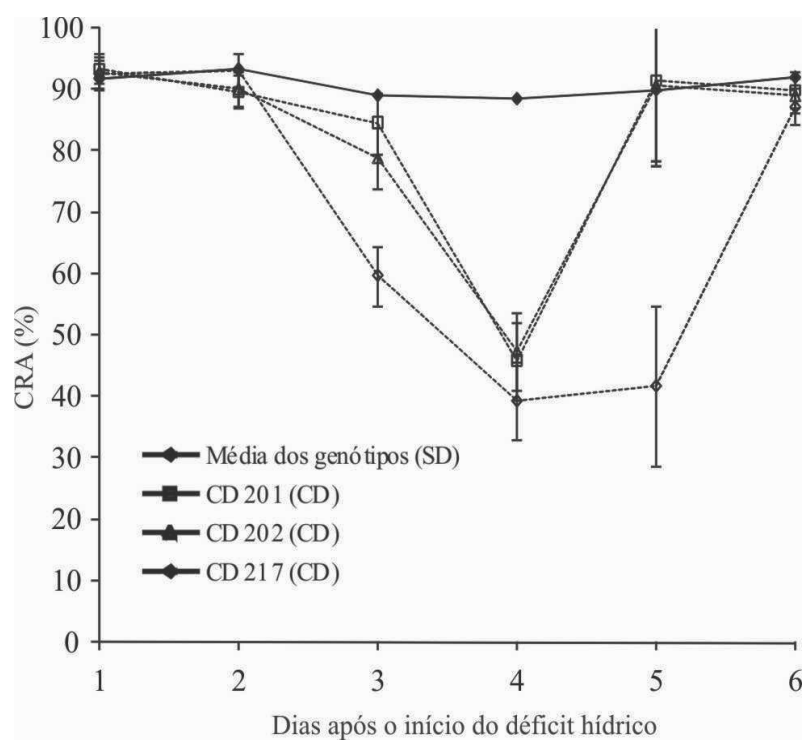

Figura 2. Conteúdo relativo de água nas folhas de três genótipos de soja submetidos a déficit hídrico intenso no início do período reprodutivo $(\mathrm{CD})$ e mantidos sob irrigação (SD). As barras verticais representam os valores de diferença mínima significativa (dms) pelo teste de Tukey a 5\% de probabilidade.

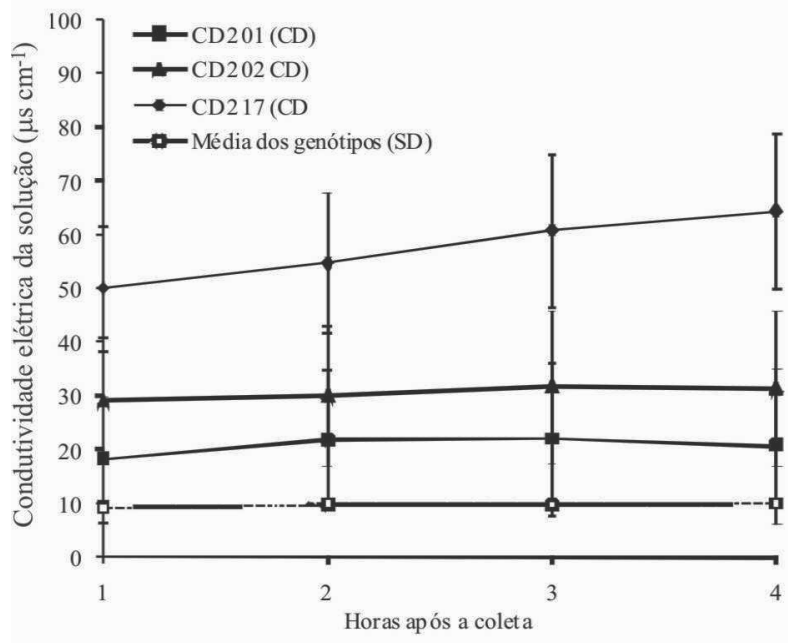

Figura 4. Condutividade elétrica de discos foliares de três genótipos de soja submetidos a déficit hídrico intenso no início do período reprodutivo. As barras verticais representam os valores de diferença mínima significativa (dms) pelo teste de Tukey a 5\% de probabilidade. 
cit hídrico, pode estar relacionada com uma maior eficiência no controle estomático da transpiração, sem haver, contudo, a necessidade de reduzir a área foliar (Blum, 2005). A manutenção da taxa de acúmulo de assimilados, bem como da área foliar, é característica importante para a manutenção do potencial produtivo de plantas em condição de déficit hídrico (Lawn \& Likoswe, 2008), apresentando variabilidade entre materiais genéticos, conforme apresentado no presente estudo.

Quando o déficit hídrico atinge condições extremas, observa-se a morte progressiva de folhas, iniciando-se pelas mais velhas e seguindo para as mais novas, quando a planta não consegue manter seu status hídrico acima do CRAc (conteúdo relativo de água crítico) (Lawn \& Likoswe, 2008). Apesar de o aumento da taxa de abscisão foliar ser uma estratégia de escape à seca, reduções drásticas na área fotossinteticamente ativa da planta podem acarretar danos significativos na produção final (O'Toole et al., 1977). Pimentel \& Cruz Perez (2000) observaram comportamento diferenciado, principalmente para a área foliar, em três experimentos conduzidos com genótipos de feijão em cultivo protegido, quando submetidos a déficit hídrico no início do florescimento. Segundo James et al. (2008b), genótipos de soja, cultivados em condições terminais de déficit hídrico, apresentaram diferenças na sobrevivência de folhas e plantas, em função do CRA. A sobrevivência de folhas e plantas foi maior em genótipos que apresentaram menores taxas de declínio no CRA e baixos valores de CRAc.

Os resultados obtidos para os componentes da produção e produção por planta (Tabela 4) refletem, claramente, o comportamento dos genótipos de soja para as relações hídricas e variáveis biométricas. O genótipo CD 217 foi superior aos demais genótipos, para número de vagens totais e vagens viáveis, demonstrando alto potencial produtivo em cultivo irrigado. Para número de grãos por planta e massa de grãos por planta, o genótipo CD 217 não diferiu do CD 201. Em condições de cultivo sob déficit hídrico, não se observaram diferenças significativas entre os genótipos. Para o genótipo CD 201, a diminuição na produção de grãos sob déficit hídrico ocorreu de forma menos acentuada. Assim como observado para as variáveis biométricas, o genótipo CD 202 não apresentou diferenças significativas entre o cultivo irrigado e com indução de déficit hídrico. A drástica diminuição do acúmulo de matéria seca, observada para o genótipo CD 217, resultou em diminuição acentuada na massa de grãos por planta, em comparação com o cultivo irrigado. Neste sentido, a manutenção do status hídrico e da área foliar em condições de déficit hídrico merece destaque, em função da sua relação com o acúmulo de assimilados e com a manutenção do potencial produtivo, conforme demonstrado no presente estudo. 
Tabela 4. Número total de vagens por planta (NTVP), número de vagens viáveis por planta (NVVP), número de grãos por planta (NGPP) e massa de grãos por planta (MGP) de genótipos de soja sob irrigação (SD) e com indução de déficit hídrico intenso no início do período reprodutivo $(\mathrm{CD})$

\begin{tabular}{|c|c|c|c|c|c|c|c|c|}
\hline \multirow[t]{2}{*}{ Genótipo } & \multicolumn{2}{|c|}{ NTVP } & \multicolumn{2}{|c|}{ NVVP } & \multicolumn{2}{|c|}{ NGPP } & \multicolumn{2}{|c|}{$\operatorname{MGP}(\mathrm{g})$} \\
\hline & SD & CD & SD & CD & SD & CD & SD & CD \\
\hline CD 201 & $123 \mathrm{bA}$ & $97 \mathrm{aB}$ & $67 \mathrm{bA}$ & $59 \mathrm{aA}$ & $130 \mathrm{aA}$ & $112 \mathrm{aA}$ & $24,9 \mathrm{aA}$ & $17,8 \mathrm{aB}$ \\
\hline CD 202 & $134 \mathrm{bA}$ & $116 \mathrm{aA}$ & $52 \mathrm{bA}$ & $55 \mathrm{aA}$ & $79 \mathrm{bA}$ & 97 abA & $16,7 \mathrm{bA}$ & $16,4 \mathrm{aA}$ \\
\hline CD 217 & $183 \mathrm{aA}$ & $115 \mathrm{aB}$ & $89 \mathrm{aA}$ & $53 \mathrm{aB}$ & $120 \mathrm{aA}$ & $76 \mathrm{bB}$ & 26,9 aA & $14,1 \mathrm{aB}$ \\
\hline dms genótipo & \multicolumn{2}{|c|}{31,15} & \multicolumn{2}{|c|}{18,97} & \multicolumn{2}{|c|}{31,75} & \multicolumn{2}{|c|}{4,71} \\
\hline dms regime & \multicolumn{2}{|c|}{25,73} & \multicolumn{2}{|c|}{15,67} & \multicolumn{2}{|c|}{26,23} & \multicolumn{2}{|c|}{3,89} \\
\hline
\end{tabular}

Médias seguidas da mesma letra, minúscula, para a comparação entre genótipos, na coluna, e maiúscula para a comparação entre regimes hídricos, na linha, não diferem estatisticamente pelo teste de Tukey a $5 \%$ de probabilidade.

Existe uma enorme vantagem, na manutenção da área foliar, em genótipos que apresentaram baixa capacidade de remobilização de reservas para os grãos, pois o acúmulo de massa passa a apresentar alta dependência da fotossíntese nas folhas (Blum, 2005). Para Pimentel et al. (2000) e Gomes et al. (2000), o rendimento final da cultura pode estar relacionado com a manutenção da área foliar durante o período de escassez de água. Portanto, a habilidade da planta em manter a área foliar, durante a diminuição da água disponível no solo, desempenha importante papel na manutenção do potencial produtivo do genótipo, após o re-estabelecimento do suprimento de água (Ludlow \& Muchow, 1990).

Características adaptativas que levam à manutenção do status hídrico, como o controle estomático, devem ser consideradas para o aumento da produtividade em condição de seca, com objetivo de habilitar a planta a competir com o estresse (Cattivelli et al., 2008). Os resultados obtidos no presente estudo permitem apontar a importância da manutenção do status hídrico, em condições de déficit hídrico, para a recuperação no período "pós-déficit", bem como para a manutenção do potencial produtivo. Devese ressaltar o comportamento do genótipo CD 202, que apresentou elevada capacidade de manter seu metabolismo, mesmo em condições de déficit hídrico de alta intensidade. Portanto, a compilação de dados, provenientes de diferentes locais e condições de cultivo, deve ser empregada para o desenvolvimento de novos materiais, com características de interesse para o cultivo em áreas com restrição hídrica.

\section{CONCLUSÕES}

A diferenciação de comportamento de genótipos de soja é possível em condição de déficit hídrico de alta intensidade e curta duração.

A manutenção do conteúdo relativo de água e da área foliar mostra-se boa estratégia para a manutenção do potencial produtivo, em genótipos de soja submetidos a déficit hídrico intenso.
Características adaptativas, como a manutenção do status hídrico e da área foliar, devem ser consideradas para a manutenção da produtividade em condições de déficit hídrico, com objetivo de habilitar a planta a competir com o estresse.

O genótipo CD 202 mostrou-se mais eficiente na manutenção do potencial produtivo, em condição de déficit hídrico de alta intensidade e curta duração, mediante a manutenção do status hídrico e a área foliar no período de estresse.

\section{REFERÊNCIAS}

Alonso A, Queiroz CGS \& Magalhães AC (1997) Chilling stress leads to increased cell membrane rigidity in roots of coffee (Coffea arabica L.) seedlings. Biochimica et Biophysica Acta, 1323:75-84

Barrs HD \& Weatherley PE (1962) A re-examination of the relative turgidity technique for estimating water deficits in leaves. Australian Journal of Biological Science, 15:413-428.

Blum A (2005) Drought resistance, water-use efficiency, and yield potential - are they compatible, dissonant, or mutually exclusive? Australian Journal of Agricultural Research, 56:1159-1168.

Cattivelli F, Rizza F, Badeck F, Mazzucotelli E, Mastrangelo AM, Francia E, Mare C, Tondelli A \& Stanca AM (2008) Drought tolerance improvement in crop plants: An integrated view from breeding to genomics. Field Crops Research, 105:1-14.

EMBRAPA. Centro Nacional de Pesquisa de Solos - CNPS (2006) Sistema brasileiro de classificação de solos. Brasília, EMBRAPASP/EMBRAPA-CNPS. 412p.

Ennahli S \& Earl HJ (2005) Physiological limitations to photosynthetic carbon assimilation in cotton under water stress. Crop Science, 45:2374-2382.

Gomes AA, Araújo AP, Rossiello ROP \& Pimentel C (2000) Acumulação de biomassa, características fisiológicas e rendimento de grãos em genótipos de feijoeiro irrigado e sob sequeiro. Pesquisa Agropecuária Brasileira, 35:1927-1937.

Gupta SC, Rathore AK, Sharma SN \& Saini RS (2000) Responses of chickpea cultivars to water stress. Indian Journal Plant Physiology, 5:274-276.

James AT, Lawn RJ \& Cooper M (2008a) Genotypic variation for drought stress response traits in soybean. I. Variation in soybean and wild Glycine spp. for epidermal conductance, osmotic potential and relative water content. Australian Journal of Agricultural Research, 59:656-669. 
James AT, Lawn RJ \& Cooper M (2008b) Genotypic variation for drought stress response traits in soybean. II. Inter-relations between epidermal conductance, osmotic potential, relative water content, and plant survival. Australian Journal of Agricultural Research, 59:670-678.

Jones HG (2007) Monitoring plant and soil water status: established and novel methods revisited and their relevance to studies of drought tolerance. Journal of Experimental Botany, 58:119130

Lawlor DW \& Uprety DC (1993) Effects of the water stress on the photosynthesis of crops and biochemical mechanism. In: Abrol YP, Mohanty P \& Govindje E (Ed.) Photosynthesis: photoreactions to plant productivity. New Dehli, Oxford and IBH Publishing Co. p.419-449.

Lawn RJ \& Likoswe AA (2008) Genotypic differences in leaf area maintenance contribute to differences in recovery from water stress in soybean. Australian Journal of Agricultural Research, 59:1075-1085

Leopold AC, Musgrave ME \& Williams KM (1981) Solute leakage resulting from leaf desiccation. Plant Physiology, 68:1222-1225.

Levitt J (1972) Responses of plants to environmental stress. New York, Academic Press. 698p.

Ludlow MM \& Muchow RC (1990) A critical evaluation of traits for improving crop yields in water-limited environments. Advances in Agronomy, 43:107-153.

O’Toole JC, Ozbun JL \& Wallace DH (1977) Photosynthetic response to water stress in Phaseoulus vulgaris. Physiologia Plantarum, 40:111-114.
Pannu RK, Singh DP, Singh P, Chaudhary BD \& Singh VP (1993) Evaluation of various plant water indices for screening the genotypes of chickpea under limited water environment. Haryana Journal Agronomy, 9:16-22.

Pimentel C \& Cruz Perez AJ (2000) Estabelecimento de parâmetros para avaliação de tolerância à seca, em genótipos de feijoeiro. Pesquisa Agropecuária Brasileira, 35:31-39.

Pimentel C, Costa ES, Dos Santos MG \& Guimarães VF (2000) Leaf protoplasmic tolerance to water stress in bean genotypes. Physiology and Molecular Biology of Plants, 6:15-20.

Pimentel C, Sarr B, Diouf O, Abboud ACS \& Roy-Macauley H (2002) Tolerância protoplasmática foliar à seca, em dois genótipos de caupi cultivados em campo. Revista Universidade Rural, Série Ciências da Vida, 22:07-14.

Roy-Macauley H, Zuily-Fodil Y, Kidric M, Phan Thi A \& Vieira da Silva J (1992) Effect of drought stress on proteolytic activities in Phaseolus and Vigna leaves from sensitive and resistant plants. Physiologia Plantarum, 85:90-96.

Sinclair TR \& Ludlow MM (1986) Influence of soil water supply on the plant water balance of four tropical grain legumes. Australian Journal of Plant Physiology, 13:329-341.

Turner NC (1997) Further progress in crop water relations. Advances in Agronomy, 58:293-338.

Voltas J, Lopez-Corcoles H \& Borras G (2005) Use of biplot analysis and factorial regression for the investigation of superior genotypes in multienvironment trials. European Journal of Agronomy, 22:309-324. 